Appl. Set-Valued Anal. Optim. 2 (2020), No. 2, pp. 255-270

Available online at http://asvao.biemdas.com

https://doi.org/10.23952/asvao.2.2020.2.09

\title{
A SHRINKING PROJECTION ALGORITHM FOR PROXIMAL SPLIT FEASIBILITY AND FIXED POINT PROBLEMS
}

\author{
JINZUO CHEN
}

School of Mathematics and Statistics, Lingnan Normal University, Zhanjiang, China

\begin{abstract}
In this paper, we study proximal split feasibility, and fixed point problems. For solving these problems, we introduce a shrinking projection algorithm in the framework of Hilbert spaces. It is proven that the sequence generated by the proposed iterative algorithm converge to a common solution of a proximal split feasibility problem and a fixed point problem of an asymptotically $k$-strictly pseudocontractive mapping in the intermediate sense.
\end{abstract}

Keywords. Asymptotically $k$-strictly pseudo-contractive mapping; Proximal split feasibility problem; Shrinking projection method; Variational inequality problem.

\section{INTRODUCTION}

Throughout this paper, we assume that $\mathscr{H}_{1}$ and $\mathscr{H}_{2}$ are two real Hilbert spaces, $A: \mathscr{H}_{1} \rightarrow \mathscr{H}_{2}$ is a bounded linear operator with its adjoint $A^{*}, f: \mathscr{H}_{1} \rightarrow \mathscr{R} \bigcup\{+\infty\}$ and $g: \mathscr{H}_{2} \rightarrow \mathscr{R} \bigcup\{+\infty\}$ are two proper, lower semi-continuous convex functions.

We study the problem of solving the following minimization problem:

$$
\min _{x \in \mathscr{H}_{1}}\left\{f(x)+g_{\lambda}(A x)\right\}
$$

where $g_{\lambda}$ stands for the Moreau-Yosida approximate of the function $g$ of index $\lambda>0$, that is,

$$
g_{\lambda}(x)=\min _{y \in \mathscr{H}_{2}}\left\{g(y)+\frac{1}{2 \lambda}\|x-y\|^{2}\right\} .
$$

Split feasibility problem [8] was introduced for modeling inverse problems, which arise from phase retrievals and in medical image reconstruction [1,9]. It was found that the split feasibility problem can also be used to model the intensity modulated therapy [6, 7]. It is also worth pointing out that the split feasibility problem is a special case of the problem (1.1). As a matter of fact, we choose $f$ and $g$ as the indicator functions of two nonempty closed convex sets $C \subset \mathscr{H}_{1}$ and $Q \subset \mathscr{H}_{2}$, that is,

$$
f(x)=\delta_{C}(x)= \begin{cases}0, & \text { if } x \in C, \\ +\infty, & \text { otherwise }\end{cases}
$$

E-mail address: chanjanegeoger@hotmail.com.

Received June 2, 2020; Accepted August 19, 2020.

(C)2020 Journal of Nonlinear Functional Analysis 
and

$$
g(x)=\delta_{Q}(x)= \begin{cases}0, & \text { if } x \in Q, \\ +\infty, & \text { otherwise }\end{cases}
$$

Then, problem (1.1) collapses to

$$
\min _{x \in \mathscr{H}_{1}}\left\{\delta_{C}(x)+\left(\delta_{Q}\right)_{\lambda}(A x)\right\},
$$

which is equivalent to the following formulation

$$
\min _{x \in C}\left\{\frac{1}{2 \lambda}\left\|\left(I-\operatorname{proj}_{Q}\right) A x\right\|^{2}\right\} .
$$

It is easy to see that solving (1.2) is to solve the following split feasibility problem of finding $x$ such that

$$
x \in C \text { and } A x \in Q
$$

provided that $C \bigcap A^{-1}(Q) \neq \emptyset$.

To solve (1.3), many authors have studied various iterative methods; see, for instance, [2, 3, $4,14,15,18,19,20,21]$. Fixed-point methods are very popular recently. It is known that a vector $x^{*}$ solves (1.3) if and only if it solves the following fixed-point equation

$$
x^{*}=\operatorname{proj}_{C}\left(I-\gamma A^{*}\left(I-\operatorname{proj}_{Q}\right) A\right) x^{*},
$$

where $\gamma>0$ is a constant and $\operatorname{proj}_{C}$ and $\operatorname{proj}_{Q}$ stand for the orthogonal projectional on the closed convex sets $C$ and $Q$, respectively. According to the above fixed point formulation, A popular algorithm that solves the split feasibility problem is the $C Q$ algorithm presented by Byrne [1,2]:

$$
x_{n+1}=\operatorname{proj}_{C}\left(x_{n}-\tau_{n} A^{*}\left(I-\operatorname{proj}_{Q}\right) A x_{n}\right),
$$

where the step size $\tau_{n} \in\left(0,2 /\|A\|^{2}\right)$. However, the step size $\tau_{n}$ depends on the operator norm $\|A\|$, which is not an easy work to calculate in practice. To overcome this difficulty, the following so-called self-adaptive method was developed recently.

Self-adaptive algorithm [11] Let $x_{0} \in \mathscr{H}_{1}$ be an initial arbitrarily point. Assume that the sequence $\left\{x_{n}\right\}$ in $C$ is constructed with $\nabla \bar{h}\left(x_{n}\right) \neq 0$ as follows: Compute $x_{n+1}$ via the rule

$$
x_{n+1}=\operatorname{proj}_{C}\left(x_{n}-\tau_{n} A^{*}\left(I-\operatorname{proj}_{Q}\right) A x_{n}\right),
$$

where $\tau_{n}=\rho_{n} \frac{\bar{h}\left(x_{n}\right)}{\left\|\nabla \bar{h}\left(x_{n}\right)\right\|^{2}}$ with $0<\rho_{n}<4$ and $\bar{h}(x)=\frac{1}{2}\left\|\left(I-\operatorname{proj}_{Q}\right) A x\right\|^{2}$.

If $\nabla \bar{h}\left(x_{n}\right)=0$, then $x_{n+1}=x_{n}$ is a solution of problem (1.3) and the iterative process stops. Otherwise, we set $n:=n+1$ and go to the sequence (1.4).

The main purpose of this manuscript is to solve problem (1.1) by using the fixed-point technique, the self-adaptive method and the shrinking projection technique. By use of the Fréchet differentiability of the Yosida approximate $g_{\lambda}$, we have

$$
\partial\left(f(x)+g_{\lambda}(A x)\right)=\partial f(x)+A^{*} \nabla g_{\lambda}(A x)=\partial f(x)+A^{*}\left(\frac{I-\operatorname{prox}_{\lambda g}}{\lambda}\right) A x,
$$

where $\partial f(x)$ denotes the subdifferential of $f$ at $x$ and $\operatorname{prox}_{\lambda g} x$ is the proximal mapping of $g$, that is,

$$
\partial f(x)=\left\{w \in \mathscr{H}_{1}: f(y) \geq f(x)+\langle w, y-x\rangle, \forall y \in \mathscr{H}_{1}\right\}
$$


and

$$
\operatorname{prox}_{\lambda g} x=\arg \min _{y \in \mathscr{H}_{2}}\left\{g(y)+\frac{1}{2 \lambda}\|y-x\|^{2}\right\} .
$$

Note that the optimality condition of (1.5) is as follows:

$$
0 \in \partial f(x)+A^{*}\left(\frac{I-\operatorname{prox}_{\lambda g}}{\lambda}\right) A x,
$$

which can be rewritten as

$$
0 \in \mu \lambda \partial f(x)+\mu A^{*}\left(I-\operatorname{prox}_{\lambda g}\right) A x
$$

This is equivalent to

$$
x=\operatorname{prox}_{\mu \lambda f}\left(I-\mu A^{*}\left(I-\operatorname{prox}_{\lambda g}\right) A\right) x
$$

for all $\mu>0$. If $\arg \min f \bigcap A^{-1}(\arg \min g) \neq \emptyset$, then (1.1) is reduced to the following proximal split feasibility problem of finding $x$ such that

$$
x \in \arg \min f \text { and } A x \in \arg \min g,
$$

where

$$
\arg \min f=\left\{x^{*} \in \mathscr{H}_{1}: f\left(x^{*}\right) \leq f(x), \forall x \in \mathscr{H}_{1}\right\}
$$

and

$$
\arg \min g=\left\{x^{\dagger} \in \mathscr{H}_{2}: f\left(x^{\dagger}\right) \leq f(x), \forall x \in \mathscr{H}_{2}\right\} .
$$

In the sequel, we use $\Gamma$ to denote the solution set of problem (1.6). Then, we have that $\Gamma$ is a convex subset of $\mathscr{H}_{1}$.

Recently, in order to solve problem (1.6), Moudafi and Thakur [14] presented the following split proximal algorithm with a way of selecting the step sizes such that its implementation does not need any prior information as regards the operator norm.

Self-adaptive split proximal algorithm [14] Let $x_{0} \in \mathscr{H}_{1}$ be an initial arbitrarily point. Assume that the sequence $\left\{x_{n}\right\}$ in $\mathscr{H}_{1}$ is constructed with $\theta\left(x_{n}\right) \neq 0$ as follows: Compute $x_{n+1}$ via the rule

$$
x_{n+1}=\operatorname{prox}_{\mu_{n} \lambda f}\left(x_{n}-\mu_{n} A^{*}\left(I-\operatorname{prox}_{\lambda g}\right) A x_{n}\right)
$$

for all $n \geq 0$, where the step size $\mu_{n}=\rho_{n} \frac{h\left(x_{n}\right)+l\left(x_{n}\right)}{\theta^{2}\left(x_{n}\right)}$ in which $0<\rho_{n}<4$,

$$
\begin{aligned}
& h\left(x_{n}\right)=\frac{1}{2}\left\|\left(I-\operatorname{prox}_{\lambda g}\right) A x_{n}\right\|^{2}, \\
& l\left(x_{n}\right)=\frac{1}{2}\left\|\left(I-\operatorname{prox}_{\mu_{n} \lambda f}\right) x_{n}\right\|^{2}
\end{aligned}
$$

and

$$
\theta\left(x_{n}\right)=\sqrt{\left\|\nabla h\left(x_{n}\right)\right\|^{2}+\left\|\nabla l\left(x_{n}\right)\right\|^{2}} .
$$

If $\theta\left(x_{n}\right)=0$, then $x_{n+1}=x_{n}$ is a solution of problem (1.6) and the iterative process stops. Otherwise, we set $n:=n+1$ and go to the sequence (1.7).

They demonstrated the following weak convergence of the above split proximal algorithm. 
Theorem 1.1. Suppose that $\Gamma \neq \emptyset$. Assume the parameters satisfy the condition:

$$
\varepsilon \leq \rho_{n} \leq \frac{4 h\left(x_{n}\right)}{h\left(x_{n}\right)+l\left(x_{n}\right)}-\varepsilon
$$

for some $\varepsilon>0$ small enough. Then the sequence $\left\{x_{n}\right\}$ generated by (1.7) weakly converges to a solutions of the problem (1.6).

They established the weak convergence of algorithm (1.7), however, in some applied disciplines, the strong convergence is more desirable than the weak convergence. So, Yao et al. [20] suggested a new algorithm by using the regularization technique such that the strong convergence is guaranteed. More precisely, they presented the following scheme:

$$
x_{n+1}=\alpha_{n} \psi\left(x_{n}\right)+\left(I-\alpha_{n} B\right) \operatorname{prox}_{\lambda f}\left(x_{n}-\mu_{n} A^{*}\left(I-\operatorname{prox}_{\lambda g}\right) A x_{n}\right)
$$

for all $n \geq 0$, where $\left\{\alpha_{n}\right\} \subset[0,1]$ is a real number sequence, $\psi: \mathscr{H}_{1} \rightarrow \mathscr{H}_{1}$ is a $\kappa$-contraction and $B: \mathscr{H}_{1} \rightarrow \mathscr{H}_{1}$ is a strongly positive bounded linear operator with coefficient $\zeta>\kappa$.

In this paper, our modification is mainly based on an idea in Takahashi, Takeuchi and Kubota [17] for finding a fixed point of a nonexpansive mapping $T$ in a Hilbert space. To be more precise, their algorithmic scheme is the following: For $u \in \mathscr{H}_{1}$ and $n \geq 1$, set

$$
\left\{\begin{array}{l}
y_{n}=\alpha_{n} x_{n}+\left(1-\alpha_{n}\right) T x_{n}, \\
C_{n+1}=\left\{w \in C_{n}:\left\|y_{n}-w\right\| \leq\left\|x_{n}-w\right\|\right\}, \\
x_{n+1}=\operatorname{proj}_{C_{n+1}} u .
\end{array}\right.
$$

Motivated by the above results, especially by Moudafi and Thakur [14], Takahashi, Takeuchi and Kubota [17], and Yao et al. [20] we introduce an iterative algorithm and prove its strong convergence for solving the proximal split feasibility problem and the fixed point problem of an asymptotically $k$-strictly pseudo-contractive mapping in the intermediate sense.

\section{PRELIMINARIES}

Let $\mathscr{H}$ be a real Hilbert space whose inner product and norm are denoted by $\langle\cdot, \cdot\rangle$ and $\|\cdot\|$, respectively. Let $C$ be a nonempty closed convex subset of $\mathscr{H}$. We write $x_{n} \rightarrow x$ to indicate that the sequence $\left\{x_{n}\right\}$ converges weakly to $x$ and $x_{n} \rightarrow x$ to indicate that the sequence $\left\{x_{n}\right\}$ converges strongly to $x$. Moreover, we use $\omega_{w}\left(x_{n}\right)$ to denote the weak $\omega$-limit set of the sequence $\left\{x_{n}\right\}$, that is,

$$
\omega_{w}\left(x_{n}\right)=\left\{x: x_{n_{i}} \rightarrow x \text { for some subsequence }\left\{x_{n_{i}}\right\} \text { of }\left\{x_{n}\right\}\right\} .
$$

The notation Fix $(T)$ denotes the set of fixed points of the mapping $T$, that is, $\operatorname{Fix}(T)=\{x \in$ $\mathscr{H}: T x=x\}$. The metric projection is an important tool in this paper. Recall that the metric (nearest point) projection from $\mathscr{H}$ onto $C$, denoted by $\operatorname{proj}_{C}$, is defined in such a way that, for each $x \in \mathscr{H}, \operatorname{proj}_{C} x$ is the unique point in $C$ with the property

$$
\left\|x-\operatorname{proj}_{C} x\right\|=\min \{\|x-y\|: y \in C\} .
$$

Some properties of projections are gathered in the following proposition.

Proposition 2.1. Given $x \in \mathscr{H}$ and $z \in C$, we have the following

(1) $z=\operatorname{proj}_{C} x \Leftrightarrow\langle x-z, y-z\rangle \leq 0$ for all $y \in C$.

(2) $z=\operatorname{proj}_{C} x \Leftrightarrow\|x-z\|^{2} \leq\|x-y\|^{2}-\|y-z\|^{2}$ for all $y \in C$. 
(3) $\left\langle x-y, \operatorname{proj}_{C} x-\operatorname{proj}_{C} y\right\rangle \geq\left\|\operatorname{proj}_{C} x-\operatorname{proj}_{C} y\right\|^{2}$ for all $y \in \mathscr{H}$, which hence implies that $\operatorname{proj}_{C}$ is nonexpansive.

Next, we give some necessary definitions for our convergence theorem.

Definition 2.1. A nonlinear operator $T: \mathscr{H} \rightarrow \mathscr{H}$ is said to be (1) L-Lipschitzian if there exists $L>0$ such that

$$
\|T x-T y\| \leq L\|x-y\|
$$

for all $x, y \in \mathscr{H}$. If $L=1, T$ is said to be nonexpansive.

(2) Firmly nonexpansive if

$$
\|T x-T y\|^{2} \leq\|x-y\|^{2}-\|(I-T) x-(I-T) y\|^{2}
$$

for all $x, y \in \mathscr{H}$, which is equivalent to

$$
\|T x-T y\|^{2} \leq\langle T x-T y, x-y\rangle
$$

for all $x, y \in \mathscr{H}$. Also, the mapping $I-T$ is firmly nonexpansive.

Note that the proximal mapping of $g$ is firmly nonexpansive, namely,

$$
\left\|\operatorname{prox}_{\lambda g} x-\operatorname{prox}_{\lambda g} y\right\|^{2} \leq\left\langle\operatorname{prox}_{\lambda g} x-\operatorname{prox}_{\lambda g} y, x-y\right\rangle
$$

for all $x, y \in \mathscr{H}_{2}$ and it is also the case for the complement $I-\operatorname{prox}_{\lambda g}$.

Definition 2.2. [16] Let $C$ be a nonempty subset of a Hilbert space $\mathscr{H}$. A mapping $T: C \rightarrow$ $C$ is said to be an asymptotically $k$-strictly pseudo-contractive mapping in the intermediate sense with sequence $\left\{\gamma_{n}\right\}$ if there exist a constant $k \in[0,1)$ and a sequence $\left\{\gamma_{n}\right\} \subset[0, \infty)$ with $\lim _{n \rightarrow \infty} \gamma_{n}=0$ such that

$$
\limsup _{n \rightarrow \infty} \sup _{x, y \in C}\left(\left\|T^{n} x-T^{n} y\right\|^{2}-\left(1+\gamma_{n}\right)\|x-y\|^{2}-k\left\|x-T^{n} x-\left(y-T^{n} y\right)\right\|^{2}\right) \leq 0 .
$$

Throughout this paper, we assume that

$$
c_{n}:=\max \left\{0, \sup _{x, y \in C}\left(\left\|T^{n} x-T^{n} y\right\|^{2}-\left(1+\gamma_{n}\right)\|x-y\|^{2}-k\left\|x-T^{n} x-\left(y-T^{n} y\right)\right\|^{2}\right)\right\} .
$$

Then $c_{n} \geq 0$ for all $n \geq 1, c_{n} \rightarrow 0$ as $n \rightarrow \infty$ and (2.1) reduces to the relation

$$
\left\|T^{n} x-T^{n} y\right\|^{2} \leq\left(1+\gamma_{n}\right)\|x-y\|^{2}+k\left\|x-T^{n} x-\left(y-T^{n} y\right)\right\|^{2}+c_{n}, \quad \forall x, y \in C, n \geq 1 .
$$

Remark 2.1. (1) $T$ is not necessarily uniformly Lipschitzian (see [16]).

(2) If $c_{n}=0$ for all $n \geq 1$ in (2.2), then $T$ is an asymptotically $k$-strictly pseudo-contractive mapping with sequence $\left\{\gamma_{n}\right\}$ (see [10]).

For all $x, y, z \in \mathscr{H}$ and $\alpha, \beta, \gamma \in[0,1]$ with $\alpha+\beta+\gamma=1$, the following conclusions are obvious:

$$
\begin{gathered}
\|\alpha x+\beta y+\gamma z\|^{2}=\alpha\|x\|^{2}+\beta\|y\|^{2}+\gamma\|z\|^{2}-\alpha \beta\|x-y\|^{2}-\alpha \gamma\|x-z\|^{2}-\beta \gamma\|y-z\|^{2} \\
\|x+y\|^{2} \leq\|x\|^{2}+2\langle y, x+y\rangle
\end{gathered}
$$

and

$$
\|x+y\|^{2}=\|x\|^{2}+2\langle x, y\rangle+\|y\|^{2} .
$$


Definition 2.3. A mapping $B: \mathscr{H} \rightarrow \mathscr{H}$ is called $\bar{\gamma}$-strongly positive, if there exists a constant $\bar{\gamma}>0$ such that

$$
\langle B x, x\rangle \geq \bar{\gamma}\|x\|^{2}, \quad \forall x \in \mathscr{H} .
$$

Lemma 2.1. [12] Assume that $B: \mathscr{H} \rightarrow \mathscr{H}$ is a $\bar{\gamma}$-strongly positive bounded linear operator on $\mathscr{H}$ with $0<\mu<\|B\|^{-1}$. Then $\|I-\mu B\| \leq 1-\mu \bar{\gamma}$.

Usually, the convergence of iterative algorithms requires some additional smoothness properties of the mapping $T$ such as demi-closedness.

Definition 2.4. An operator $T$ is said to be demi-closed iffor any sequence $\left\{x_{n}\right\}$, which weakly converges to $x$, and if the sequence $\left\{T x_{n}\right\}$ strongly converges to $z$, then $T x=z$.

Lemma 2.2. [16] (Demiclosedness principle) Let $C$ be a nonempty closed convex subset of a Hilbert space $\mathscr{H}$ and $T: C \rightarrow C$ be a continuous asymptotically $k$-strictly pseudo-contractive mapping in the intermediate sense. Then $I-T$ is demiclosed at zero in the sense that if $\left\{x_{n}\right\}$ is a sequence in $C$ such that $x_{n} \rightarrow x \in C$ and $\limsup _{m \rightarrow \infty} \limsup _{n \rightarrow \infty}\left\|x_{n}-T^{m} x_{n}\right\|=0$, then $(I-T) x=0$.

Lemma 2.3. [16] Let $C$ be a nonempty subset of a Hilbert space $\mathscr{H}$ and $T: C \rightarrow C$ be an asymptotically $k$-strictly pseudo-contractive mapping in the intermediate sense with sequence $\left\{\gamma_{n}\right\}$. Then

$$
\left\|T^{n} x-T^{n} y\right\| \leq \frac{1}{1-k}\left(k\|x-y\|+\sqrt{\left(1+\gamma_{n}(1-k)\right)\|x-y\|^{2}+c_{n}(1-k)}\right)
$$

for all $x, y \in C$ and $n \geq 1$.

Lemma 2.4. [16] Let $C$ be a nonempty subset of a Hilbert space $\mathscr{H}$ and $T: C \rightarrow C$ be a uniformly continuous asymptotically $k$-strictly pseudo-contractive mapping in the intermediate sense with sequence $\left\{\gamma_{n}\right\}$. Let $\left\{x_{n}\right\}$ be a sequence in $C$ such that $\left\|x_{n}-x_{n+1}\right\| \rightarrow 0$ and $\| x_{n}-$ $T^{n} x_{n} \| \rightarrow 0$ as $n \rightarrow \infty$. Then $\left\|x_{n}-T x_{n}\right\| \rightarrow 0$ as $n \rightarrow \infty$.

Lemma 2.5. [16] Let $C$ be a nonempty closed convex subset of a Hilbert space $\mathscr{H}$ and $T: C \rightarrow$ $C$ be a continuous asymptotically $k$-strictly pseudo-contractive mapping in the intermediate sense. Then Fix $(T)$ is closed and convex.

Lemma 2.6. [16] Let $\mathscr{H}$ be a real Hilbert space. Given a nonempty closed convex subset $C$ of $\mathscr{H}$ and points $x, y, z \in \mathscr{H}$ and given also a real number $a \in \mathbb{R}$, the set

$$
\left\{w \in C:\|y-w\|^{2} \leq\|x-w\|^{2}+\langle z, w\rangle+a\right\}
$$

is convex (and closed).

Lemma 2.7. [13] Let $C$ be a nonempty closed convex subset of a real Hilbert $\mathscr{H}$ and let $\left\{x_{n}\right\}$ be a sequence in $\mathscr{H}$ and $x_{0} \in \mathscr{H}$. Let $q=P_{C} x_{0}$. If $\left\{x_{n}\right\}$ satisfies the following conditions: (1) $\omega_{w}\left(x_{n}\right) \subset C$; (2) $\left\|x_{n}-x_{0}\right\| \leq\left\|x_{0}-q\right\|$ for all $n \geq 1$, then $x_{n} \rightarrow q$. 


\section{MAIN RESULT}

In this section, we introduce the iterative algorithm and prove its strong convergence.

Assume that $\mathscr{H}_{1}$ and $\mathscr{H}_{2}$ are two real Hilbert spaces, $f: \mathscr{H}_{1} \rightarrow \mathscr{R} \bigcup\{+\infty\}$ and $g: \mathscr{H}_{2} \rightarrow$ $\mathscr{R} \bigcup\{+\infty\}$ are two proper, lower semi-continuous convex functions and that (1.6) is consistent. $A: \mathscr{H}_{1} \rightarrow \mathscr{H}_{2}$ is a bounded linear operator with its adjoint $A^{*}$. Let $C$ be a nonempty closed convex subset of a real Hilbert $\mathscr{H}_{1}$. Assume that $\psi: \mathscr{H}_{1} \rightarrow \mathscr{H}_{1}$ is a contraction with coefficient $\eta \in(0,1)$. Let $T: C \rightarrow C$ be a uniformly continuous asymptotically $k$-strictly pseudo-contractive mapping in the intermediate sense with $\left\{\gamma_{n}\right\}$ and let $c_{n}$ be defined as in (2.1). Let $B: \mathscr{H}_{1} \rightarrow \mathscr{H}_{1}$ is a strongly positive bounded linear operator with coefficient $\bar{\gamma}$. Set $\theta(x)=\sqrt{\|\nabla h(x)\|^{2}+\|\nabla l(x)\|^{2}}$ with $h(x)=\frac{1}{2}\left\|\left(I-\operatorname{prox}_{\lambda g}\right) A x\right\|^{2}, l(x)=\frac{1}{2}\left\|\left(I-\operatorname{prox}_{\mu_{n} \lambda f}\right) x\right\|^{2}$ for all $x \in \mathscr{H}_{1}$ and introduce the following algorithm.

Algorithm 3.1. For an initialization $x_{0}, x_{1} \in \mathscr{H}_{1}$, assume that $\left\{x_{n}\right\}$ is a sequence generated by the rule with $\theta\left(x_{n}\right) \neq 0$,

$$
\left\{\begin{array}{l}
y_{n}=\operatorname{prox}_{\mu_{n} \lambda f}\left(x_{n}-\mu_{n} A^{*}\left(I-\operatorname{prox}_{\lambda g}\right) A x_{n}\right), \\
z_{n}=\beta_{n} y_{n}+\sigma_{n} \mu \psi\left(y_{n}\right)+\left(\left(1-\beta_{n}\right) I-\sigma_{n} B\right) T^{n} y_{n}, \\
C_{n+1}=\left\{w \in C_{n}:\left\|z_{n}-w\right\|^{2} \leq\left\|x_{n}-w\right\|^{2}+\tau_{n}\right\}, \\
x_{n+1}=\operatorname{proj}_{C_{n+1}} x_{0},
\end{array}\right.
$$

where $\Delta_{n}=\sup \left\{\left\|x_{n}-x^{*}\right\|^{2}+\frac{\mu}{1-\eta}\left\|\psi\left(x^{*}\right)-x^{*}\right\|^{2}+\left\|\mu x^{*}-B x^{*}\right\|^{2}: x^{*} \in F i x(T) \bigcap \Gamma\right\}<\infty$ for all $n \geq 1$, and $\tau_{n}=\left(1-\sigma_{n}(1+\mu-\bar{\gamma})\right)^{-1}\left(c_{n}+\left(\sigma_{n}(1+\mu-\bar{\gamma})+\gamma_{n}\right) \Delta_{n}\right)$. Assume that $\mu_{n}$ is the step size satisfying $\mu_{n}=\rho_{n} \frac{h\left(x_{n}\right)+l\left(x_{n}\right)}{\theta^{2}\left(x_{n}\right)}$ with $0<\rho_{n}<4,\left\{\beta_{n}\right\},\left\{\sigma_{n}\right\} \subset[0,1]$ are two real number sequences and $\mu>0$ is a constant.

If $\theta\left(x_{n}\right)=0$, then $x_{n+1}=x_{n}$ is a solution of problem (1.6), which is also a fixed point of a uniformly continuous asymptotically $k$-strictly pseudo-contractive mapping in the intermediate sense and the iterative process stops. Otherwise, we set $n:=n+1$ and go to the sequence (3.1).

We are now in a position to show our main convergence theorem.

Theorem 3.1. Suppose that Fix $(T) \cap \Gamma$ is nonempty and bounded and the parameters satisfy the condition:

(i) $\varepsilon \leq \rho_{n} \leq \frac{4 h\left(x_{n}\right)}{h\left(x_{n}\right)+l\left(x_{n}\right)}-\varepsilon$ for some $\varepsilon>0$ small enough;

(ii) $\lim _{n \rightarrow \infty} \sigma_{n}=0$;

(iii) $\beta_{n}+\sigma_{n} \mu \leq 1$;

(iv) $0 \leq k<\beta_{n}\left(1-\beta_{n}-\sigma_{n} \mu\right)$;

(v) $\bar{\gamma}<\mu<\bar{\gamma} / \eta$.

Then the sequence $\left\{x_{n}\right\}$ generated by (3.1) strongly converges to $p_{0}=P_{F i x(T) \cap \Gamma} x_{0}$, which is the unique solution in Fix $(T) \cap \Gamma$ to the following variational inequality:

$$
\left\langle(B-\mu \psi) p_{0}, x^{*}-p_{0}\right\rangle \geq 0, \quad \forall x^{*} \in F i x(T) \bigcap \Gamma,
$$

provided that $\left\|x_{n}-z_{n}\right\|=\circ\left(\sigma_{n}\right)$ and $\gamma_{n}+c_{n}+\left\|T^{n} y_{n}-y_{n}\right\|^{2}=\circ\left(\sigma_{n}\right)$. Equivalently,

$$
p_{0}=P_{F i x(T) \cap \Gamma}(I-B+\mu \psi) p_{0} .
$$

Proof. Let $x^{*} \in \operatorname{Fix}(T) \cap \Gamma$. Since the minimizers of any function are exactly fixed points of its proximal mappings, we have $x^{*}=\operatorname{prox}_{\mu_{n} \lambda f} x^{*}$ and $A x^{*}=\operatorname{prox}_{\lambda g} A x^{*}$. Using the fact that 
$\operatorname{prox}_{\mu_{n} \lambda f}$ is nonexpansive, we derive from (3.1) that

$$
\begin{aligned}
\left\|y_{n}-x^{*}\right\| & =\left\|\operatorname{prox}_{\mu_{n} \lambda f}\left(x_{n}-\mu_{n} A^{*}\left(I-\operatorname{prox}_{\lambda g}\right) A x_{n}\right)-x^{*}\right\| \\
& \leq\left\|x_{n}-\mu_{n} A^{*}\left(I-\operatorname{prox}_{\lambda g}\right) A x_{n}-x^{*}\right\| .
\end{aligned}
$$

Note that

$$
\nabla h\left(x_{n}\right)=A^{*}\left(I-\operatorname{prox}_{\lambda g}\right) A x_{n}
$$

and

$$
\nabla l\left(x_{n}\right)=\left(I-\operatorname{prox}_{\mu_{n} \lambda f}\right) x_{n}
$$

Since $I-\operatorname{prox}_{\lambda g}$ is firmly nonexpansive, we obtain

$$
\begin{aligned}
& \left\|x_{n}-\mu_{n} A^{*}\left(I-\operatorname{prox}_{\lambda g}\right) A x_{n}-x^{*}\right\|^{2} \\
= & \left\|x_{n}-x^{*}\right\|^{2}-2 \mu_{n}\left\langle A^{*}\left(I-\operatorname{prox}_{\lambda g}\right) A x_{n}, x_{n}-x^{*}\right\rangle+\mu_{n}^{2}\left\|A^{*}\left(I-\operatorname{prox}_{\lambda g}\right) A x_{n}\right\|^{2} \\
\leq & \left\|x_{n}-x^{*}\right\|^{2}-4 \mu_{n} h\left(x_{n}\right)+\mu_{n}^{2}\left\|\nabla h\left(x_{n}\right)\right\|^{2} \\
\leq & \left\|x_{n}-x^{*}\right\|^{2}-\frac{4 \rho_{n}\left(h\left(x_{n}\right)+l\left(x_{n}\right)\right)^{2}}{\theta^{2}\left(x_{n}\right)} \frac{h\left(x_{n}\right)}{h\left(x_{n}\right)+l\left(x_{n}\right)}+\frac{\rho_{n}^{2}\left(h\left(x_{n}\right)+l\left(x_{n}\right)\right)^{2}}{\theta^{2}\left(x_{n}\right)} \\
= & \left\|x_{n}-x^{*}\right\|^{2}-\frac{\rho_{n}\left(h\left(x_{n}\right)+l\left(x_{n}\right)\right)^{2}}{\theta^{2}\left(x_{n}\right)}\left(\frac{4 h\left(x_{n}\right)}{h\left(x_{n}\right)+l\left(x_{n}\right)}-\rho_{n}\right) .
\end{aligned}
$$


By virtue of (2.2), (2.3), (2.4), (3.1), conditions (iii), (v), Lemma 2.1 and the definition of $T$, we conclude that

$$
\begin{aligned}
& \left\|z_{n}-x^{*}\right\|^{2} \\
& =\left\|\beta_{n} y_{n}+\sigma_{n} \mu \psi\left(y_{n}\right)+\left(\left(1-\beta_{n}\right) I-\sigma_{n} B\right) T^{n} y_{n}-x^{*}\right\|^{2} \\
& \leq\left\|\beta_{n} y_{n}+\sigma_{n} \mu \psi\left(y_{n}\right)+\left(1-\beta_{n}-\sigma_{n} \mu\right) T^{n} y_{n}-x^{*}\right\|^{2} \\
& +2\left\langle\left(\left(1-\beta_{n}\right) I-\sigma_{n} B\right) T^{n} y_{n}-\left(1-\beta_{n}-\sigma_{n} \mu\right) T^{n} y_{n}, z_{n}-x^{*}\right\rangle \\
& \leq \beta_{n}\left\|y_{n}-x^{*}\right\|^{2}-\beta_{n}\left(1-\beta_{n}-\sigma_{n} \mu\right)\left\|y_{n}-T^{n} y_{n}\right\|^{2}+\left(1-\beta_{n}-\sigma_{n} \mu\right)\left\|T^{n} y_{n}-x^{*}\right\|^{2} \\
& +\sigma_{n} \mu\left\|\psi\left(y_{n}\right)-x^{*}\right\|^{2}+2 \sigma_{n}\left\langle(\mu I-B)\left(T^{n} y_{n}-x^{*}\right)+(\mu I-B) x^{*}, z_{n}-x^{*}\right\rangle \\
& \leq \beta_{n}\left\|y_{n}-x^{*}\right\|^{2}+\sigma_{n} \mu\left(\left\|\psi\left(y_{n}\right)-\psi\left(x^{*}\right)\right\|+\left\|\psi\left(x^{*}\right)-x^{*}\right\|\right)^{2} \\
& +\left(1-\beta_{n}-\sigma_{n} \mu\right)\left\|T^{n} y_{n}-x^{*}\right\|^{2}-\beta_{n}\left(1-\beta_{n}-\sigma_{n} \mu\right)\left\|y_{n}-T^{n} y_{n}\right\|^{2} \\
& +2 \sigma_{n}\left(\|\mu I-B\|\left\|T^{n} y_{n}-x^{*}\right\|\left\|z_{n}-x^{*}\right\|+\left\|\mu x^{*}-B x^{*}\right\|\left\|z_{n}-x^{*}\right\|\right) \\
& \leq \beta_{n}\left\|y_{n}-x^{*}\right\|^{2}+\sigma_{n} \mu\left(\eta\left\|y_{n}-x^{*}\right\|+(1-\eta) \frac{1}{1-\eta}\left\|\psi\left(x^{*}\right)-x^{*}\right\|\right)^{2} \\
& +\left(1-\beta_{n}-\sigma_{n} \mu\right)\left\|T^{n} y_{n}-x^{*}\right\|^{2}-\beta_{n}\left(1-\beta_{n}-\sigma_{n} \mu\right)\left\|y_{n}-T^{n} y_{n}\right\|^{2} \\
& +\sigma_{n}\left((\mu-\bar{\gamma})\left(\left\|T^{n} y_{n}-x^{*}\right\|^{2}+\left\|z_{n}-x^{*}\right\|^{2}\right)+\left(\left\|\mu x^{*}-B x^{*}\right\|^{2}+\left\|z_{n}-x^{*}\right\|^{2}\right)\right) \\
& =\left(\beta_{n}+\sigma_{n} \mu \eta\right)\left\|y_{n}-x^{*}\right\|^{2}+\frac{\sigma_{n} \mu}{1-\eta}\left\|\psi\left(x^{*}\right)-x^{*}\right\|^{2} \\
& +\left(1-\beta_{n}-\sigma_{n} \mu+\sigma_{n}(\mu-\bar{\gamma})\right)\left\|T^{n} y_{n}-x^{*}\right\|^{2}-\beta_{n}\left(1-\beta_{n}-\sigma_{n} \mu\right)\left\|y_{n}-T^{n} y_{n}\right\|^{2} \\
& +\sigma_{n}(1+\mu-\bar{\gamma})\left\|z_{n}-x^{*}\right\|^{2}+\sigma_{n}\left\|\mu x^{*}-B x^{*}\right\|^{2} \\
& \leq\left(\beta_{n}+\sigma_{n} \mu \eta\right)\left\|y_{n}-x^{*}\right\|^{2}+\frac{\sigma_{n} \mu}{1-\eta}\left\|\psi\left(x^{*}\right)-x^{*}\right\|^{2}-\beta_{n}\left(1-\beta_{n}-\sigma_{n} \mu\right)\left\|y_{n}-T^{n} y_{n}\right\|^{2} \\
& +\left(1-\beta_{n}-\sigma_{n} \mu+\sigma_{n}(\mu-\bar{\gamma})\right)\left(\left(1+\gamma_{n}\right)\left\|y_{n}-x^{*}\right\|^{2}+k\left\|y_{n}-T^{n} y_{n}\right\|^{2}+c_{n}\right) \\
& +\sigma_{n}(1+\mu-\bar{\gamma})\left\|z_{n}-x^{*}\right\|^{2}+\sigma_{n}\left\|\mu x^{*}-B x^{*}\right\|^{2} \\
& \leq\left(\beta_{n}+\sigma_{n} \bar{\gamma}+\left(1-\beta_{n}-\sigma_{n} \bar{\gamma}\right)\left(1+\gamma_{n}\right)\right)\left\|y_{n}-x^{*}\right\|^{2}+\frac{\sigma_{n} \mu}{1-\eta}\left\|\psi\left(x^{*}\right)-x^{*}\right\|^{2} \\
& +\left(1-\beta_{n}-\sigma_{n} \bar{\gamma}\right)\left(k-\beta_{n} \frac{1-\beta_{n}-\sigma_{n} \mu}{1-\beta_{n}-\sigma_{n} \bar{\gamma}}\right)\left\|y_{n}-T^{n} y_{n}\right\|^{2} \\
& +\left(1-\beta_{n}-\sigma_{n} \bar{\gamma}\right) c_{n}+\sigma_{n}(1+\mu-\bar{\gamma})\left\|z_{n}-x^{*}\right\|^{2}+\sigma_{n}\left\|\mu x^{*}-B x^{*}\right\|^{2} \\
& \leq\left(1+\gamma_{n}\right)\left\|y_{n}-x^{*}\right\|^{2}+\frac{\sigma_{n} \mu}{1-\eta}\left\|\psi\left(x^{*}\right)-x^{*}\right\|^{2}+c_{n}+\sigma_{n}(1+\mu-\bar{\gamma})\left\|z_{n}-x^{*}\right\|^{2} \\
& +\left(1-\beta_{n}-\sigma_{n} \bar{\gamma}\right)\left(k-\beta_{n}\left(1-\beta_{n}-\sigma_{n} \mu\right)\right)\left\|y_{n}-T^{n} y_{n}\right\|^{2}+\sigma_{n}\left\|\mu x^{*}-B x^{*}\right\|^{2} \\
& \leq\left(1+\gamma_{n}\right)\left(\left\|x_{n}-x^{*}\right\|^{2}-\frac{\rho_{n}\left(h\left(x_{n}\right)+l\left(x_{n}\right)\right)^{2}}{\theta^{2}\left(x_{n}\right)}\left(\frac{4 h\left(x_{n}\right)}{h\left(x_{n}\right)+l\left(x_{n}\right)}-\rho_{n}\right)\right) \\
& +\frac{\sigma_{n} \mu}{1-\eta}\left\|f\left(x^{*}\right)-x^{*}\right\|^{2}+\left(1-\beta_{n}-\sigma_{n} \bar{\gamma}\right)\left(k-\beta_{n}\left(1-\beta_{n}-\sigma_{n} \mu\right)\right)\left\|y_{n}-T^{n} y_{n}\right\|^{2} \\
& +c_{n}+\sigma_{n}(1+\mu-\bar{\gamma})\left\|z_{n}-x^{*}\right\|^{2}+\sigma_{n}\left\|\mu x^{*}-B x^{*}\right\|^{2} \text {. }
\end{aligned}
$$


Without loss of generality, by use of the control condition $(i)$, we can assume that

$$
\left(4 h\left(x_{n}\right)\right) /\left(h\left(x_{n}\right)+l\left(x_{n}\right)\right)-\rho_{n} \geq 0
$$

for all $n \geq 1$. Thus, from (3.2), (3.3), (3.4) and the conditions (iii $-v)$, we have

$$
\begin{aligned}
\left\|z_{n}-x^{*}\right\|^{2} \leq & \left(1+\gamma_{n}\right)\left\|x_{n}-x^{*}\right\|^{2}+\frac{\sigma_{n} \mu}{1-\eta}\left\|\psi\left(x^{*}\right)-x^{*}\right\|^{2}+\sigma_{n}(1+\mu-\bar{\gamma})\left\|z_{n}-x^{*}\right\|^{2} \\
& +c_{n}+\sigma_{n}\left\|\mu x^{*}-B x^{*}\right\|^{2} .
\end{aligned}
$$

This implies that

$$
\begin{aligned}
& \left(1-\sigma_{n}(1+\mu-\bar{\gamma})\right)\left\|z_{n}-x^{*}\right\|^{2} \\
\leq & \left(1+\gamma_{n}\right)\left\|x_{n}-x^{*}\right\|^{2}+\frac{\sigma_{n} \mu}{1-\eta}\left\|\psi\left(x^{*}\right)-x^{*}\right\|^{2}+c_{n}+\sigma_{n}\left\|\mu x^{*}-B x^{*}\right\|^{2} .
\end{aligned}
$$

From the conditions $(i i)$ and $(v)$, we obtain

$$
\begin{aligned}
\left\|z_{n}-x^{*}\right\|^{2} \leq & \left\|x_{n}-x^{*}\right\|^{2}+\frac{1}{1-\sigma_{n}(1+\mu-\bar{\gamma})}\left(\left(\sigma_{n}(1+\mu-\bar{\gamma})+\gamma_{n}\right)\left\|x_{n}-x^{*}\right\|^{2}\right. \\
& \left.+\left(\sigma_{n} \mu /(1-\eta)\right)\left\|\psi\left(x^{*}\right)-x^{*}\right\|^{2}+c_{n}+\sigma_{n}\left\|\mu x^{*}-B x^{*}\right\|^{2}\right) \\
\leq & \left\|x_{n}-x^{*}\right\|^{2}+\frac{1}{1-\sigma_{n}(1+\mu-\bar{\gamma})}\left(( \sigma _ { n } ( 1 + \mu - \overline { \gamma } ) + \gamma _ { n } ) \left(\left\|x_{n}-x^{*}\right\|^{2}\right.\right. \\
& \left.\left.+(\mu /(1-\eta))\left\|\psi\left(x^{*}\right)-x^{*}\right\|^{2}+\left\|\mu x^{*}-B x^{*}\right\|^{2}\right)+c_{n}\right) \\
\leq & \left\|x_{n}-x^{*}\right\|^{2}+\frac{1}{1-\sigma_{n}(1+\mu-\bar{\gamma})}\left(\left(\sigma_{n}(1+\mu-\bar{\gamma})+\gamma_{n}\right) \Delta_{n}+c_{n}\right) \\
= & \left\|x_{n}-x^{*}\right\|^{2}+\tau_{n},
\end{aligned}
$$

where

$$
\Delta_{n}=\sup \left\{\left\|x_{n}-x^{*}\right\|^{2}+\frac{\mu}{1-\eta}\left\|\psi\left(x^{*}\right)-x^{*}\right\|^{2}+\left\|\mu x^{*}-B x^{*}\right\|^{2}: x^{*} \in F i x(T) \bigcap \Gamma\right\}<\infty,
$$

and

$$
\tau_{n}=\left(1-\sigma_{n}(1+\mu-\bar{\gamma})\right)^{-1}\left(\left(\sigma_{n}(1+\mu-\bar{\gamma})+\gamma_{n}\right) \Delta_{n}+c_{n}\right) .
$$

It follows that $x^{*} \in C_{n+1}$. Thus, $\operatorname{Fix}(T) \cap \Gamma \subset C_{n}$ for all $n \geq 1$.

Next, we show that $C_{n}$ is closed and convex for all $n \geq 1$. The set $C_{1}=\mathscr{H}_{1}$ is obviously closed and convex. Suppose that $C_{k}$ is closed and convex. We see that $C_{k+1}$ is closed and convex since $\left\|z_{n}-w\right\|^{2} \leq\left\|x_{n}-w\right\|^{2}+\tau_{n}$ is equivalent to

$$
2\left\langle x_{n}-z_{n}, w\right\rangle \leq\left\|x_{n}\right\|^{2}-\left\|z_{n}\right\|^{2}+\tau_{n} .
$$

From Lemma 2.6, we have $C_{n}$ is closed and convex for all $n \geq 1$. Thus, we obtain that the sequence $\left\{x_{n}\right\}$ is well defined. From Proposition 2.1(1) and $x_{n}=P_{C_{n}} x_{0}$, we have

$$
\left\langle x_{0}-x_{n}, x_{n}-y\right\rangle \geq 0, \quad \forall y \in C_{n} .
$$

Recalling that $F i x(T) \bigcap \Gamma \subset C_{n}$, one has

$$
\left\langle x_{0}-x_{n}, x_{n}-x^{*}\right\rangle \geq 0, \quad \forall x^{*} \in F i x(T) \bigcap \Gamma .
$$


Hence,

$$
\begin{aligned}
0 & \leq\left\langle x_{0}-x_{n}, x_{n}-x^{*}\right\rangle \\
& =\left\langle x_{0}-x_{n}, x_{n}-x_{0}+x_{0}-x^{*}\right\rangle \\
& \leq-\left\|x_{n}-x_{0}\right\|^{2}+\left\|x_{n}-x_{0}\right\|\left\|x_{0}-x^{*}\right\| .
\end{aligned}
$$

This implies that

$$
\left\|x_{n}-x_{0}\right\| \leq\left\|x_{0}-x^{*}\right\|
$$

which yields that sequence $\left\{x_{n}\right\}$ is bounded. We can also easy to get $\left\{y_{n}\right\},\left\{z_{n}\right\},\left\{\psi\left(y_{n}\right)\right\}$ and $\left\{T^{n} y_{n}\right\}$ are bounded. From $x_{n}=P_{C_{n}} x_{0}$ and $x_{n+1}=P_{C_{n+1}} x_{0} \in C_{n+1} \subset C_{n}$, we get

$$
\begin{aligned}
0 & \leq\left\langle x_{0}-x_{n}, x_{n}-x_{n+1}\right\rangle \\
& \leq-\left\|x_{n}-x_{0}\right\|^{2}+\left\|x_{n}-x_{0}\right\|\left\|x_{0}-x_{n+1}\right\|,
\end{aligned}
$$

which gives that

$$
\left\|x_{n}-x_{0}\right\| \leq\left\|x_{0}-x_{n+1}\right\|
$$

Hence, the $\operatorname{limit}_{n \rightarrow \infty}\left\|x_{n}-x_{0}\right\|$ exists. It follows from (3.7) that

$$
\begin{aligned}
\left\|x_{n}-x_{n+1}\right\|^{2} & =\left\|x_{n}-x_{0}\right\|^{2}+2\left\langle x_{n}-x_{0}, x_{0}-x_{n+1}\right\rangle+\left\|x_{0}-x_{n+1}\right\|^{2} \\
& =-\left\|x_{n}-x_{0}\right\|^{2}+2\left\langle x_{n}-x_{0}, x_{n}-x_{n+1}\right\rangle+\left\|x_{0}-x_{n+1}\right\|^{2} \\
& \leq-\left\|x_{n}-x_{0}\right\|^{2}+\left\|x_{0}-x_{n+1}\right\|^{2} .
\end{aligned}
$$

Thus,

$$
\lim _{n \rightarrow \infty}\left\|x_{n}-x_{n+1}\right\|=0 .
$$

The fact that $x_{n+1}=P_{C_{n+1}} x_{0} \in C_{n+1}$ gives

$$
\left\|z_{n}-x_{n+1}\right\|^{2} \leq\left\|x_{n}-x_{n+1}\right\|^{2}+\tau_{n} \leq\left(\left\|x_{n}-x_{n+1}\right\|+\sqrt{\tau_{n}}\right)^{2} .
$$

In view of condition $(i i)$ and the fact that $\lim _{n \rightarrow \infty} c_{n}=0, \lim _{n \rightarrow \infty} \gamma_{n}=0$, we have $\lim _{n \rightarrow \infty} \tau_{n}=0$. The expressions (3.8) and (3.9) yield

$$
\lim _{n \rightarrow \infty}\left\|z_{n}-x_{n}\right\|=0
$$

Returning to (3.4) and (3.5), we have

$$
\begin{aligned}
& \left(1+\gamma_{n}\right)\left(\frac{\rho_{n}\left(h\left(x_{n}\right)+l\left(x_{n}\right)\right)^{2}}{\theta^{2}\left(x_{n}\right)}\left(\frac{4 h\left(x_{n}\right)}{h\left(x_{n}\right)+l\left(x_{n}\right)}-\rho_{n}\right)\right) \\
& +\left(1-\beta_{n}-\sigma_{n} \bar{\gamma}\right)\left(k-\beta_{n}\left(1-\beta_{n}-\sigma_{n} \mu\right)\right)\left\|y_{n}-T^{n} y_{n}\right\|^{2} \\
\leq & \left(1+\gamma_{n}\right)\left(\left\|x_{n}-x^{*}\right\|^{2}-\left\|z_{n}-x^{*}\right\|^{2}\right)+\frac{\sigma_{n} \mu}{1-\eta}\left\|f\left(x^{*}\right)-x^{*}\right\|^{2}+c_{n} \\
& +\left(1+\gamma_{n}-\left(1-\sigma_{n}(1+\mu-\bar{\gamma})\right)\right)\left\|z_{n}-x^{*}\right\|^{2}+\sigma_{n}\left\|\mu x^{*}-B x^{*}\right\|^{2} \\
= & \left(1+\gamma_{n}\right)\left(\left\|x_{n}-x^{*}\right\|+\left\|z_{n}-x^{*}\right\|\right)\left\|z_{n}-x_{n}\right\|+\frac{\sigma_{n} \mu}{1-\eta}\left\|f\left(x^{*}\right)-x^{*}\right\|^{2}+c_{n} \\
& +\left(\gamma_{n}+\sigma_{n}(1+\mu-\bar{\gamma})\right)\left\|z_{n}-x^{*}\right\|^{2}+\sigma_{n}\left\|\mu x^{*}-B x^{*}\right\|^{2} .
\end{aligned}
$$


This, together with (3.10), conditions $(i i-v), \rho_{n}\left(\frac{4 h\left(x_{n}\right)}{h\left(x_{n}\right)+l\left(x_{n}\right)}-\rho_{n}\right) \geq \varepsilon^{2}, \lim _{n \rightarrow \infty} c_{n}=0$ and $\lim _{n \rightarrow \infty} \gamma_{n}=0$, implies that

$$
\lim _{n \rightarrow \infty} \frac{\left(h\left(x_{n}\right)+l\left(x_{n}\right)\right)^{2}}{\theta^{2}\left(x_{n}\right)}=0
$$

and

$$
\lim _{n \rightarrow \infty}\left\|y_{n}-T^{n} y_{n}\right\|=0 .
$$

Noting that $\theta^{2}\left(x_{n}\right)=\left\|\nabla h\left(x_{n}\right)\right\|^{2}+\left\|\nabla l\left(x_{n}\right)\right\|^{2}$ is bounded, we deduce from (3.11) immediately that

$$
\lim _{n \rightarrow \infty}\left(h\left(x_{n}\right)+l\left(x_{n}\right)\right)=0 .
$$

Therefore,

$$
\lim _{n \rightarrow \infty} h\left(x_{n}\right)=\lim _{n \rightarrow \infty} l\left(x_{n}\right)=0 .
$$

Since $\left\{x_{n}\right\}$ is bounded, there exists a subsequence $\left\{x_{n_{i}}\right\}$ of $\left\{x_{n}\right\}$ such that $x_{n_{i}} \rightarrow p$. By the lower semi-continuity of $h$, we have

$$
0 \leq h(p) \leq \liminf _{i \rightarrow \infty} h\left(x_{n_{i}}\right)=\lim _{n \rightarrow \infty} h\left(x_{n}\right)=0,
$$

which implies

$$
h(p)=\frac{1}{2}\left\|\left(I-\operatorname{prox}_{\lambda g}\right) A p\right\|=0,
$$

that is, $A p$ is a fixed point of proximal mapping of $g$ or, equivalently, $0 \in \partial g(A p)$. In other words, $A p$ is a minimizer of $g$.

Similarly, from the lower semi-continuity of $l$, we have

$$
0 \leq l(p) \leq \liminf _{i \rightarrow \infty} l\left(x_{n_{i}}\right)=\lim _{n \rightarrow \infty} l\left(x_{n}\right)=0 .
$$

Hence,

$$
l(p)=\frac{1}{2}\left\|\left(I-\operatorname{prox}_{\mu_{n} \lambda f}\right) p\right\|=0,
$$

that is, $p$ is a fixed point of the proximal mapping of $f$ or, equivalently, $0 \in \partial f(p)$. In other words, $p$ is a minimizer of $f$. Hence, $p \in \Gamma$.

We observe that $0<\mu_{n}<\frac{4\left(h\left(x_{n}\right)+l\left(x_{n}\right)\right)}{\theta^{2}\left(x_{n}\right)}$, which implies that $\lim _{n \rightarrow \infty} \mu_{n}=0$. Hence, we have from (3.1) and the boundedness of $\theta\left(x_{n}\right)$ that

$$
\left\|y_{n}-\operatorname{prox}_{\mu_{n} \lambda f} x_{n}\right\| \leq \mu_{n}\left\|A^{*}\left(I-\operatorname{prox}_{\lambda g}\right) A x_{n}\right\| \leq \mu_{n} M
$$

for some $M>0$. From $l\left(x_{n}\right)=\frac{1}{2}\left\|\left(I-\operatorname{prox}_{\mu_{n} \lambda f}\right) x_{n}\right\|^{2}$, we have

$$
\lim _{n \rightarrow \infty}\left\|\left(I-\operatorname{prox}_{\mu_{n} \lambda f}\right) x_{n}\right\|=0 \text {. }
$$

This together with (3.14) implies that

$$
\lim _{n \rightarrow \infty}\left\|y_{n}-x_{n}\right\|=0 .
$$


Furthermore, using Lemma 2.3, we have

$$
\begin{aligned}
\left\|x_{n}-T^{n} x_{n}\right\| \leq & \left\|x_{n}-y_{n}\right\|+\left\|y_{n}-T^{n} y_{n}\right\|+\left\|T^{n} y_{n}-T^{n} x_{n}\right\| \\
\leq & \left\|x_{n}-y_{n}\right\|+\left\|y_{n}-T^{n} y_{n}\right\|+\frac{1}{1-\kappa}\left(k\left\|y_{n}-x_{n}\right\|\right. \\
& +\sqrt{\left.\left(1+\gamma_{n}(1-k)\right)\left\|y_{n}-x_{n}\right\|^{2}+c_{n}(1-k)\right)} .
\end{aligned}
$$

From (3.12), (3.15) and (3.16), we get

$$
\lim _{n \rightarrow \infty}\left\|x_{n}-T^{n} x_{n}\right\|=0
$$

By Lemma 2.4, we obtain

$$
\lim _{n \rightarrow \infty}\left\|x_{n}-T x_{n}\right\|=0
$$

Since $T$ is uniformly continuous and by (3.18), it is easy to obtain that $\lim _{n \rightarrow \infty}\left\|x_{n}-T^{m} x_{n}\right\|=0$ for all $m \geq 1$. From Lemma 2.2, we have $p \in \operatorname{Fix}(T)$. So, $p \in F i x(T) \cap \Gamma$. Thus, $\omega_{w}\left(x_{n}\right) \in$ $\operatorname{Fix}(T) \cap \Gamma$. According to Lemma 2.5 and Lemma 2.7, we see that $x_{n} \rightarrow p_{0}=P_{F i x(T) \cap \Gamma x_{0}}$ as $n \rightarrow \infty$.

Furthermore, we assume additionally that $\left\|x_{n}-z_{n}\right\|=\circ\left(\sigma_{n}\right)$ and $\gamma_{n}+c_{n}+\left\|T^{n} y_{n}-y_{n}\right\|^{2}=$ $\circ\left(\sigma_{n}\right)$. We next show that $p_{0}$ is the unique solution in $\operatorname{Fix}(T) \cap \Gamma$ to the following variational inequality:

$$
\left\langle(B-\mu \psi) p_{0}, x^{*}-p_{0}\right\rangle \geq 0, \quad \forall x^{*} \in \operatorname{Fix}(T) \bigcap \Gamma
$$

Equivalently, $p_{0}=P_{F i x(T) \cap \Gamma}(I-B+\mu \psi) p_{0}$. Indeed, by the definition of $B$ and $\psi$, for all $x, y \in$ $\mathscr{H}_{1}$, we have

$$
\begin{aligned}
\langle(B-\mu \psi) x-(B-\mu \psi) y, x-y\rangle & =\langle B x-B y, x-y\rangle-\mu\langle\psi(x)-\psi(y), x-y\rangle \\
& \geq(\bar{\gamma}-\mu \eta)\|x-y\|^{2} .
\end{aligned}
$$

This implies that $B-\mu \psi$ is $(\bar{\gamma}-\mu \eta)$-strongly monotone. In the meantime, we obtain

$$
\|(B-\mu \psi) x-(B-\mu \psi) y\| \leq\|B x-B y\|+\mu\|\psi(x)-\psi(y)\| \leq(\|B\|+\mu \eta)\|x-y\| .
$$

That is to say, $B-\mu \psi$ is $(\|B\|+\mu \eta)$-Lipschitz continuous. Thus, there exists a unique solution $\widehat{p} \in \operatorname{Fix}(T) \bigcap \Gamma$ to satisfy the following variational inequality:

$$
\left\langle(B-\mu \psi) \widehat{p}, x^{*}-\widehat{p}\right\rangle \geq 0, \quad \forall x^{*} \in F i x(T) \bigcap \Gamma
$$


Equivalently, $\widehat{p}=P_{F i x(T) \cap \Gamma}(I-B+\mu \psi) \widehat{p}$. By (2.2), (2.3), (2.4), (3.1), (3.2), (3.3) and conditions (iii), $(v)$, we obtain

$$
\begin{aligned}
\left\|z_{n}-x^{*}\right\|^{2}= & \left\|\beta_{n} y_{n}+\sigma_{n} \mu \psi\left(y_{n}\right)+\left(\left(1-\beta_{n}\right) I-\sigma_{n} B\right) T^{n} y_{n}-x^{*}\right\|^{2} \\
\leq & \left\|\beta_{n}\left(y_{n}-x^{*}\right)+\sigma_{n} \mu\left(\psi\left(y_{n}\right)-\psi\left(x^{*}\right)\right)+\left(\left(1-\beta_{n}\right) I-\sigma_{n} B\right)\left(T^{n} y_{n}-x^{*}\right)\right\|^{2} \\
& +2 \sigma_{n}\left\langle(\mu \psi-B) x^{*}, z_{n}-x^{*}\right\rangle \\
\leq & \left(\beta_{n}+\sigma_{n} \bar{\gamma}\right)\left\|y_{n}-x^{*}\right\|^{2}+\left(1-\beta_{n}-\sigma_{n} \bar{\gamma}\right)\left\|T^{n} y_{n}-x^{*}\right\|^{2} \\
& +2 \sigma_{n}\left\langle(\mu \psi-B) x^{*}, z_{n}-x^{*}\right\rangle \\
\leq & \left(\beta_{n}+\sigma_{n} \bar{\gamma}\right)\left\|y_{n}-x^{*}\right\|^{2}+2 \sigma_{n}\left\langle(\mu \psi-B) x^{*}, z_{n}-x^{*}\right\rangle \\
& +\left(1-\beta_{n}-\sigma_{n} \bar{\gamma}\right)\left(\left(1+\gamma_{n}\right)\left\|y_{n}-x^{*}\right\|^{2}+k\left\|y_{n}-T^{n} y_{n}\right\|^{2}+c_{n}\right) \\
= & \left\|y_{n}-x^{*}\right\|^{2}+\left(1-\beta_{n}-\sigma_{n} \bar{\gamma}\right)\left(\gamma_{n}\left\|y_{n}-x^{*}\right\|^{2}+k\left\|y_{n}-T^{n} y_{n}\right\|^{2}+c_{n}\right) \\
& +2 \sigma_{n}\left\langle(\mu \psi-B) x^{*}, z_{n}-x^{*}\right\rangle \\
\leq & \left\|x_{n}-x^{*}\right\|^{2}+\left(\gamma_{n}+\left\|y_{n}-T^{n} y_{n}\right\|^{2}+c_{n}\right)\left(\left\|y_{n}-x^{*}\right\|^{2}+k+1\right) \\
& +2 \sigma_{n}\left\langle(\mu \psi-B) x^{*}, z_{n}-x^{*}\right\rangle,
\end{aligned}
$$

which yields

$$
\begin{aligned}
& \left\langle(\mu \psi-B) x^{*}, x^{*}-z_{n}\right\rangle \\
\leq & \frac{1}{2 \sigma_{n}}\left(\left\|x_{n}-x^{*}\right\|^{2}-\left\|z_{n}-x^{*}\right\|^{2}+\left(\gamma_{n}+\left\|y_{n}-T^{n} y_{n}\right\|^{2}+c_{n}\right)\left(\left\|y_{n}-x^{*}\right\|^{2}+k+1\right)\right) \\
\leq & \frac{\left\|x_{n}-z_{n}\right\|}{2 \sigma_{n}}\left(\left\|x_{n}-x^{*}\right\|+\left\|z_{n}-x^{*}\right\|\right) \\
& +\frac{\gamma_{n}+\left\|y_{n}-T^{n} y_{n}\right\|^{2}+c_{n}}{2 \sigma_{n}}\left(\left\|y_{n}-x^{*}\right\|^{2}+k+1\right) .
\end{aligned}
$$

By use of (3.10), (3.17) and condition (v), we can assume that $\left\|x_{n}-z_{n}\right\|=\circ\left(\sigma_{n}\right)$ and

$$
\gamma_{n}+c_{n}+\left\|T^{n} y_{n}-y_{n}\right\|^{2}=\circ\left(\sigma_{n}\right) .
$$

Since $x_{n} \rightarrow p_{0}$ as $n \rightarrow \infty$, we deduce from (3.20) that

$$
\left\langle(B-\mu \psi) x^{*}, x^{*}-p_{0}\right\rangle \geq 0, \quad \forall x^{*} \in \operatorname{Fix}(T) \bigcap \Gamma,
$$

which together with Minty's lemma (see [5]) implies that

$$
\left\langle(B-\mu \psi) p_{0}, x^{*}-p_{0}\right\rangle \geq 0, \quad \forall x^{*} \in \operatorname{Fix}(T) \bigcap \Gamma .
$$

This shows that $p_{0}$ is a solution in $\operatorname{Fix}(T) \cap \Gamma$ to the variational inequality (3.19). Using the uniqueness of solutions in $\operatorname{Fix}(T) \cap \Gamma$ of the variational inequality (3.19), we obtain that $p_{0}=$ $\widehat{p}$.

Remark 3.1. We make the following remarks concerning our contributions in this paper.

(1) Yao et al. [20, Theorem 3.2] proved strong convergence theorems for the proximal split feasibility problem by the regularization method, however, in this paper, the shrinking projection method is presented for solving the proximal split feasibility and fixed point problems.

(2) The technique in proving the strong convergence in Theorem 3.1 is different from those in Yao et al. [20, Theorem 3.2], Moudafi and Thakur [14, Theorem 2.2] since different tools, 
Lemmas 2.2-Lemma 2.7 are involved.

(3) To ensure the weak convergence of the algorithm proposed by Moudafi and Thakur [14, Theorem 2.2], one has to use the Opial's property of Hilbert spaces. The main advantage of our algorithm is that its convergence does not rely on the Opial's property. Furthermore, we establish the norm convergence of the proposed algorithm.

(4) In Theorem 3.1, we consider the problem of $\operatorname{Fix}(T) \cap \Gamma$, which is more general than the problems in Yao et al. [20, Theorem 3.2] and Moudafi and Thakur [14, Theorem 2.2].

\section{Acknowledgments}

The author is grateful to the reviewers for useful suggestions which improved the contents of this paper. This work was supported by the Ph.D. research startup foundation of Lingnan Normal University (ZL1919) and the Natural Science Foundation of Guangdong Province under grant 2018A0303070012.

\section{REFERENCES}

[1] C. Byrne, A unified treatment of some iterative algorithm in signal processing and image reconstruction, Inverse Probl. 20 (2004), 103-120.

[2] C. Byrne, Iterative oblique projection onto convex subsets and the split feasibility problem, Inverse Probl. 18 (2002), 441-453.

[3] L.C. Ceng, Q.H. Ansari, J.C. Yao Relaxed extragradient methods for finding minimum-norm solutions of the split feasibility problem, Nonlinear Anal. 75 (2012), 2116-2125.

[4] J.Z. Chen, L.C. Ceng, Y.Q. Qiu, Z.R. Kong, Extra-gradient methods for solving split feasibility and fixed point problems, Fixed Point Theory Appl. 2015 (2015), Article ID 192.

[5] R.W. Cottle, J.C. Yao, Pseudo-monotone complementarity problems in Hilbert space, J. Optim. Theory Appl. 75 (1992), 281-295.

[6] Y. Censor, A. Motova, A. Segal, Perturbed projections and subgradient projections for the multiple-sets split feasibility problem, J. Math. Anal. Appl. 327 (2007), 1244-1256.

[7] Y. Censor, S. Petra, C. Schnörr, Superiorization vs. accelerated convex optimization: The superiorized / regularized least-squares case, J. Appl. Numer. Optim. 2 (2020), 15-62.

[8] Y. Censor, T. Elfving, A multiprojection algorithm using Bregman projections in a product space, Numer. Algorithm. 8 (1994), 221-239.

[9] T. Humphries, M. Loreto, B. Halter, W. O'Keeffe, L. Ramirez, Comparison of regularized and superiorized methods for tomographic image reconstruction, J. Appl. Numer. Optim. 2 (2020), 77-99.

[10] T.H. Kim, H.K. Xu, Convergence of the modified Mann's iteration method for asymptotically strict pseudocontractions, Nonlinear Anal. 68 (2008), 2828-2836.

[11] G. Lopez, V. Martin-Marquez, F. Wang, H.K. Xu, Solving the split feasibility problem without prior knowledge of matrix norms, Inverse Probl. 28 (2012), Article ID 085004.

[12] G. Marino, H.K. Xu, A general iterative method for nonexpansive mappings in Hilbert spaces, J. Math. Anal. Appl. 318 (2006), 43-52.

[13] M. Yanes, H.K. Xu, Strong convergence of the CQ method for fixed point iteration processes, Nonlinear. Anal. 64 (2006), 2400-2411.

[14] A. Moudafi, B.S. Thakur, Solving proximal split feasibility problems without prior knowledge of operator norms, Optim. Lett. 8 (2014), 2099-2110.

[15] X. Qin, J.C. Yao, A viscosity iterative method for a split feasibility problem, J. Nonlinear Convex Anal. 20 (2019), 1497-1506.

[16] D.R. Sahu, H.K. Xu, J.C. Yao, Asymptotically strict pseudocontractive mappings in the intermediate sense Nonlinear Analysis, Theory, Methods and Applications, 70 (2009), 3502-3511.

[17] W. Takahashi, Y. Takeuchi, R. Kubota, Strong convergence theorems by hybrid methods for families of nonexpansive mappings in Hilbert spaces, J. Math. Anal. Appl. 341(2008), 276-286. 
[18] F. Wang, H.K. Xu, Approximating curve and strong convergence of the CQ algorithm for the split feasibility problem, J. Inequal. Appl. 2010 (2010), Article ID 102085.

[19] H.K. Xu, A variable Krasnosel'skii-Mann algorithm and the multiple-set split feasibility problem, Inverse Probl. 22(2006), 2021-2034.

[20] Y. Yao, Z. Yao, A.A. Abdou, Y.J. Cho, Self-adaptive algorithms for proximal split feasibility problems and strong convergence analysis, Fixed Point Theory Appl. 2015 (2015), Article ID 205.

[21] Y. Yao, M. Postolache, Y. Liou, Strong convergence of a self-adaptive method for the split feasibility problem, Fixed Point Theory Appl. 2013 (2013), Article ID 201. 